\title{
ÓRFÃOS DE TERAPIA MEDICAMENTOSA: A ADMINISTRAÇÃO DE MEDICAMENTOS POR VIA INTRAVENOSA EM CRIANÇAS HOSPITALIZADAS ${ }^{1}$
}

\author{
Maria Angélica Sorgini Peterlini \\ Massae Noda Chaud ${ }^{3}$ \\ Mavilde da L.G. Pedreira ${ }^{4}$
}

Peterlini MAS, Chaud MN, Pedreira MLG. Órfãos de terapia medicamentosa: a administração de medicamentos por via intravenosa em crianças hospitalizadas. Rev Latino-am Enfermagem 2003 janeiro-fevereiro; 11(1):88-95.

Estudo descritivo, realizado em um hospital universitário, que verificou quantidade e tipo de medicamentos administrados por via intravenosa em crianças, além da adequação da apresentação farmacológica para uso em pediatria e custo estimado de administração de algumas drogas. Em trinta dias, foram administradas 8.245 doses de medicamentos, com média diária de 274,83 doses, e projeção anual de 98.940. Os principais medicamentos utilizados foram metilpredinosolona, vancomicina, furosemida, ranitidina, penicilina, amicacina, midazolan, fentanil, ceftriaxone e cefalotina. Nenhum dos 41 medicamentos identificados possuía apresentação pediátrica, acarretando, em alguns casos, maior manipulação durante o preparo, risco de contaminação e perda da estabilidade. Observou-se que a falta de apresentação pediátrica gerou aumento dos custos de atendimento; na prescrição de uma criança em pós-operatório, com tempo de internação estimado de cinco dias, a terapia administrada diária foi de US\$6.71, e US\$39.52 de medicamentos tiveram que ser desprezados, por excederem as necessidades terapêuticas da criança.

DESCRITORES: enfermagem pediátrica, infusões, criança

\section{DRUG THERAPY ORPHANS: THE ADMINISTRATION OF INTRAVENOUS} DRUGS IN HOSPITALIZED CHILDREN

Descriptive study, developed at a general university hospital that aimed at verifying the number and types of IV drugs administered to children, the adequacy of their pharmacological presentation for pediatric use and the estimated costs of some drugs administration. In a period of 30 days, 8,245 drug doses were administered, with an average of 274.83 doses a day, and a yearly estimation of 98.940. The most used drugs were methylprednisolone, vancomycin, furosemide, ranitidine, penicillin, amikacin, midazolam, fentanyl, ceftriaxone, cephalothin, oxacillin, ampicillin and metronidazole. None of the 41 different drugs had a pediatric presentation, what caused, in some cases, more manipulation during the preparation, increasing the contamination risks and the loss of stability. Authors observed that the lack of pediatric presentation generated an increase in care costs; as an example, considering the prescription of a child in the period after surgery, with an estimated time of hospitalization of 5 days, the daily therapy costs were of $U \$ 6.71$, and $U \$ 39.52$ of drugs were thrown away as they exceeded the children therapeutic needs.

DESCRIPTORS: pediatric nursing, infusions, children

HUÉRFANOS DE TERAPIA MEDICAMENTOSA: LA ADMINISTRACIÓN DE MEDICAMENTOS POR VÍA INTRAVENOSA EN NIÑOS HOSPITALIZADOS

Estudio descriptivo realizado en un hospital de enseñanza donde se verificó la cantidad y el tipo de medicamentos administrados por vía intravenosa en niños, además de la adecuación de la presentación farmacológica para el uso en pediatría. En treinta días fueron administradas 8.245 dosis de medicamentos, con un promedio diario de 274,83 dosis y una proyección anual de 98.940. Los medicamentos más utilizados fueron metilpredinosolona, vancomicina, furosemida, ranitidina, penicilina, amicacina, midazolan, fentanil y ceftriaxone y cefalotina. Ninguno de los 41 medicamentos poseía presentación pediátrica, acarreando en una mayor manipulación, riesgo de contaminación y pérdida de estabilidad. Se observó que la falta de presentación pediátrica generó aumento en los costos de atención; en la prescripción de un niño en postoperatorio, con un tiempo de internación estimado de cinco días, la terapia administrada diaria fue de U\$6.71 y U\$ 39.52 de medicamentos que tuvieron de ser descartados por exceder las necesidades terapéuticas de los niños.

DESCRIPTORES: enfermería pediátrica, infusiones, niños

\footnotetext{
${ }^{1}$ Trabalho realizado com apoio do FADA- Fundo de Auxílio aos Docentes e Alunos da Universidade Federal de São Paulo; ${ }^{2}$ Enfermeira, Mestre em Enfermagem Pediátrica, Professor Assistente, Doutoranda em Enfermagem, e-mail: peterlini@denf.epm.br; ${ }^{3}$ Enfermeira, Doutor em Enfermagem, Professor Adjunto, e-mail: massae@denf.epm.br. Disciplina de Enfermagem Pediátrica do Departamento de Enfermagem da Universidade Federal de São Paulo
} 
INTRODUÇÃO

Para o tratamento de crianças portadoras de doenças agudas e crônicas, a administração de medicamentos por via intravenosa evidencia, cada vez mais, destacada relevância clínica.

A importância desse procedimento na, prática diária da enfermagem, foi verificada em estudo prospectivo onde, aproximadamente, dois terços das atividades diárias eram destinadas à realização de atribuições relacionadas à terapia intravenosa, que abrangiam desde 0 planejamento da administração até a retirada de cateteres intravenosos ${ }^{(1)}$.

A terapia intravenosa caracteriza-se como multidisciplinar, porém, na sua execução, as funções da enfermeira pediatra apresentam contínuos e desafiantes avanços, relacionados, principalmente, aos diferentes métodos e períodos de administração, formas e agentes utilizados para a diluição, e problemas relacionados à incompatibilidade medicamentosa, decorrente tanto da associação de drogas, como de drogas e soluções ${ }^{(2)}$.

Além desses aspectos, já detectados na década de oitenta e que continuam caracterizando-se como atuais e carentes de pesquisas nacionais em enfermagem pediátrica, soma-se, ainda, importante consideração, capaz de causar impacto tanto na assistência como nos custos dos serviços de saúde, no que se refere à falta de formulações de drogas intravenosas pediátricas ${ }^{(3)}$.

Características de absorção, distribuição, metabolismo e excreção de drogas, diferem do recémnascido ao adolescente, sendo, portanto, necessário que enfermeiras-pediatras possuam conhecimentos científicos e técnicos específicos que possibilitem a realização segura e eficaz da terapia intravenosa.

Aproximadamente $80 \%$ das drogas comercializadas para adultos não são destinadas para uso em pediatria, porém essa falta de indicação não significa necessariamente que a droga seja ineficaz, perigosa ou contra-indicada para uso em crianças ${ }^{(3)}$.

Observa-ses quotidianamente, que muitas dessas drogas são utilizadas em crianças, incluindo recémnascidos, sendo esse uso respaldado, principalmente, pela prática clínica e por pesquisas na área.

Todavia, apesar do uso freqüente e da eficácia terapêutica comprovada, não se observam transformações no mercado da industria farmacêutica, a fim de que as formulações dessas drogas atendam às necessidades específicas da criança.

O alerta publicado em 1997, pelo National Institute of Child Health and Human Development, dos Estados Unidos da América, informava que, apenas cinco das 80 drogas mais utilizadas em recém-nascidos e lactentes eram aprovadas para uso pediátrico ${ }^{(4)}$.

Uma das mais importantes implicações práticas desse fato refere-se à falta de medicamentos direcionados para aquela faixa etária, tanto no que diz respeito à forma, como à concentração e à quantidade de droga comercializada, levando alguns autores a classificarem crianças como órfãs de terapia medicamentosa ${ }^{(5)}$.

Acreditamos que, igualmente, os profissionais que trabalham na área, também se encontram desamparados, pois necessitam saber como utilizar, diluir, estocar e administrar drogas, muitas vezes sem o respaldo de evidências científicas, o que leva à constatação de diferentes práticas realizadas em serviços de atendimento pediátrico e até mesmo em diferentes setores de um mesmo hospital.

A necessidade de administrarem-se doses muito fracionadas de drogas, como, por exemplo, diluirem-se 1000 miligramas $(\mathrm{mg})$ de determinada medicação para administrarem-se $40 \mathrm{mg}$, ou aspirarem-se 8 microgramas ( $\mathrm{mcg}$ ) de outra, cuja apresentação é de $500 \mathrm{mcg}$ por mililitro de solução, geram maior demanda de tempo de trabalho de enfermagem, além da necessidade de manipulação exagerada das soluções, o que pode comprometer a qualidade do procedimento em vários aspectos, como quanto à estabilidade e possibilidade de contaminação.

Somam-se a essas dificuldades a possibilidade de intoxicação decorrente do uso de medicamentos comercializados em altas concentrações. Drogas intravenosas como morfina e fenobarbital, comercializadas para uso em adultos, apresentam alta concentração, dificultando a medida acurada de pequenas doses necessárias a recém-nascidos e lactentes, sendo que, na literatura, são relatados casos de intoxicação grave decorrente do uso da morfina ${ }^{(3)}$.

Um método utilizado para contornar esse problema, realizado na prática clínica, é a rediluição de drogas comercializadas em dose muito concentrada. Porém, questionamentos surgem, quanto à estabilidade da droga após nova diluição, esterilidade, presença de pirógenos e tempo de estocagem até a administração.

Apesar das possibilidades de riscos que advêm 
dessa prática, sabe-se que tal conduta continuará a ser realizada até que o mercado farmacêutico disponibilize medicamentos com características de formulação e apresentação direcionadas para a faixa etária pediátrica, assim facilitando e permitindo uma administração mais segura e eficaz.

O contexto exposto fundamentou a decisão em implementar um estudo que demonstre de forma quantitativa a abrangência do emprego da terapia intravenosa em crianças hospitalizadas, bem como identifique as drogas mais utilizadas, a fim de se analisar sua adequação, do ponto de vista da apresentação farmacológica, para o uso na faixa etária pediátrica e o impacto na assistência e nos custos de atendimento hospitalar.

\section{OBJETIVOS}

Os objetivos deste estudo, sobre a administração de medicamentos por via intravenosa, em crianças internadas em quatro unidades pediátricas de um hospital geral de ensino, foram:

- Verificar o número de medicamentos administrados.

- Identificar e classificar farmacologicamente os medicamentos administrados por via intravenosa mais utilizados.

- Verificar a adequação da apresentação farmacológica para o uso em pediatria.

- Estimar o custo da administração de algumas drogas utilizadas para terapia intravenosa em crianças.

\section{METODOLOGIA}

Estudo descritivo, realizado em quatro unidades pediátricas de um hospital universitário de 720 leitos da cidade de São Paulo.

Antes do início da coleta de dados, o projeto de pesquisa foi submetido à apreciação do comitê de ética em pesquisa do local de estudo, recebendo parecer favorável.

A coleta dos dados foi realizada por meio de investigação das medicações administradas por via intravenosa, registradas nas prescrições médicas diárias das crianças hospitalizadas, num período pré-estabelecido de trinta dias.

Fizeram parte da coleta de dados as prescrições de crianças internadas nas unidades: enfermaria de pediatria clínica, enfermaria de pediatria cirúrgica, enfermaria de infectologia pediátrica e unidade de cuidados intensivos pediátricos (UCIP).

Foi utilizado um protocolo de pesquisa para registro das variáveis referentes à identificação da unidade, identificação da prescrição, tipo de medicamento administrado, número de doses administradas por dia, número de profissionais de enfermagem escalados para realizar atribuições referentes à administração intravenosa de medicamentos, número diário de crianças submetidas a terapia intravenosa e apresentação farmacológica dos medicamentos administrados.

Os dados obtidos foram apurados em planilhas eletrônicas e analisados segundo freqüências absoluta e relativa, e média aritmética, sendo apresentados em tabelas.

\section{RESULTADOS}

Obteve-se uma população de 1212 prescrições e média diária, nas quatro unidades investigadas, de 40,4 crianças submetidas a terapia intravenosa.

Tabela 1 - Unidades pediátricas de um hospital de ensino, segundo número de doses de medicamentos administrados por via intravenosa e de prescrições médicas. SP, 2000

\begin{tabular}{|c|c|c|c|c|}
\hline \multirow{2}{*}{$\begin{array}{l}\text { Tipo de } \\
\text { Unidade }\end{array}$} & \multicolumn{2}{|c|}{ Número total de doses } & \multicolumn{2}{|c|}{ Número total de prescrições } \\
\hline & $\mathrm{n}$ & $\%$ & $\mathrm{n}$ & $\%$ \\
\hline Enfermaria Clínica & 3965 & 48,1 & 656 & 54,1 \\
\hline UCIP & 2297 & 27,8 & 202 & 16,7 \\
\hline Enfermaria Cirúrgica & 1029 & 12,5 & 126 & 10,4 \\
\hline Infectologia Pediátrica & 954 & 11,6 & 228 & 18,8 \\
\hline Total & 8245 & 100,0 & 1212 & 100,0 \\
\hline
\end{tabular}


Foram administradas 8.245 doses de medicamentos em 30 dias, com média de 274,83 doses/ dia e 6,8 doses/criança/dia. A maioria (75,9\%) das doses de medicamentos foram administradas na enfermaria clínica e UCIP.

Proporcionalmente ao número de prescrições, na unidade de pediatria cirúrgica, administrou-se maior número de doses, do que na infectologia pediátrica.

Tabela 2 - Unidades pediátricas de um hospital de ensino, segundo média de doses de medicamentos administrados por via intravenosa por dia e por criança/dia. SP, 2000

\begin{tabular}{ccc}
\hline Tipo de & $\begin{array}{c}\text { Dose de } \\
\text { medicamentos/dia }\end{array}$ & $\begin{array}{c}\text { Dose de } \\
\text { medicamentos/ } \\
\text { criança/dia }\end{array}$ \\
\cline { 2 - 3 } Unidade & Média & Média \\
\hline Enfermaria Clínica & 132,2 & 6,0 \\
UCIP & 76,6 & 11,4 \\
Enfermaria Cirúrgica & 34,3 & 8,2 \\
Infectologia & 31,8 & 4,2 \\
Pediátrica &
\end{tabular}

Conforme a Tabela 2, foram administrados, na unidade de pediatria clínica, maiores números de doses diárias, porém, quanto ao número de doses por pacientes, obteve-se maior número na UCIP e unidade cirúrgica, 11,4 e 8,2, respectivamente.

Na Tabela 3, são apresentados os dados obtidos referentes ao número de profissionais de enfermagem, que, no período de 30 dias, estiveram envolvidos diariamente na execução de atividades relacionadas à administração de medicamentos por via intravenosa.

Tabela 3 - Unidades pediátricas de um hospital de ensino, segundo média do número de profissionais/paciente/dia. SP, 2000

\begin{tabular}{cc}
\hline Tipo de unidade & Profissional/Paciente/dia \\
\cline { 2 - 2 } Pediátrica & média \\
\hline UCIP & 3,13 \\
Infectologia Pediátrica & 1,21 \\
Enfermaria Clínica & 1,20 \\
Enfermaria Cirúrgica & 0,95 \\
\hline
\end{tabular}

Visto que, no hospital investigado, a equipe de enfermagem trabalha em três turnos diários, obteve-se proporção aproximada entre profissional/paciente/período de 1:1 na UCIP, 1:2,5 na infectologia pediátrica, 1:2,5 na enfermaria clínica e 1:3,0 na cirúrgica. A correlação entre número de profissionais de enfermagem, segundo maior demanda de força de trabalho relacionada à terapia intravenosa não parece ter relação, principalmente, com o número de profissionais de enfermagem por criança, identificado na enfermaria cirúrgica, que obteve a menor proporção, apesar de ser a segunda unidade em doses de medicamentos/criança.

Quanto aos tipos de medicamentos identificados, obtivemos que a unidade de pediatria clínica apresentou maior número de tipos de fármacos, seguida da UCIP, infectologia e enfermaria cirúrgica, respectivamente 34, 28, 15 e 14. Foram identificados, nas quatro unidades investigadas, um total de 41 diferentes tipos de medicamentos administrados.

Verificou-se grande variação do número de doses (1 a 483) segundo tipo de medicamento, fator este relacionado à maior abrangência de emprego de alguns fármacos, bem como ação e finalidade terapêutica de cada tipo.

Os medicamentos administrados são apresentados a seguir, segundo classificação farmacológica:

- Antibióticos: amicacina, ampicilina, anfotericina b, cefalotina, cefepime, ceftazidima, ceftriaxone, cefotaxima clindamicina, cloranfenicol, emipenen, fluconazol, ganciclovir, metronidazol, oxacilina, penicilina, sulfametaoxazol trimetropim e vancomicina.

- Analgésicos opiáceos: fentanil, tramadol e meperidina.

- Benzodiazepínicos/ Anticonvulsivantes: midazolan; diazepam; fenitoína; fenobarbital sódico.

- Broncodilatadores: terbultalina; aminofilina.

- Corticóides: metilpredinosolona, hidrocortisona.

- Sintomáticos: dimenidrinato, dipirona, metoclopramida. - Vasoativos/cardiotônicos: lanatosídeo c; dobutamina, dopamina e adrenalina.

- Outros: bicarbonato de sódio, heparina, furosemida e ranitidina.

Na unidade de pediatria clínica, os medicamentos mais administrados foram: solumedrol (483 doses), vancomicina (408 doses), furosemida (328 doses), ranitidina ( 325 doses), penicilina ( 286 doses) e amicacina (242 doses).

Na UCIP, foram infundidos, com maior freqüência: midazolan (206 doses), ranitidina (203 doses), vancomicina (201 doses), amicacina (153 doses) e fentanil (146 doses).

$\mathrm{Na}$ unidade de infectologia, ministraram-se, principalmente, antibióticos como vancomicina (124 doses), 
oxacilina (106 doses), ceftriaxone (104 doses), ampicilina

(74 doses) e amicacina (68 doses).

Igualmente, na enfermaria cirúrgica, os principais medicamentos aplicados foram os antibióticos do tipo vancomicina (149 doses), amicacina (128 doses), cefalotina (100 doses) e metronidazol (88 doses), além de ranitidina ( 78 doses) e furosemida ( 41 doses).
A partir desse levantamento, selecionaram-se, dentre as drogas mais administradas em cada unidade, as que seriam pesquisadas segundo apresentação, custos e dose pediátrica recomendada, sendo estas: metilpredinisolona, vancomicina, furosemida, ranitidina, penicilina, amicacina, midazolan, fentanil, ceftriaxone, cefalotina, oxacilina, ampicilina e metronidazol.

Tabela 4 - Medicamentos mais administrados por via intravenosa, em unidades pediátricas de um hospital de ensino, apresentação farmacológica, custos em dólares americanos e dose pediátrica recomendada. SP, 2000

\begin{tabular}{cccc}
\hline Tipo de medicamento & Apresentação & Custos U\$ & Dose pediátrica \\
\hline Metilpredinosolona & Frasco $500 \mathrm{mg} \mathrm{em} 6 \mathrm{ml}$ & 22.25 & $0,4-1,6 \mathrm{mg} / \mathrm{Kg} / \mathrm{dia}$ \\
Vancomicina & Frasco $500 \mathrm{mg} \mathrm{em} 5 \mathrm{ml}$ & 13.60 & $30 \mathrm{mg} / \mathrm{Kg} / \mathrm{dia}$ \\
Furosemida & Ampola $20 \mathrm{mg} \mathrm{em} 2 \mathrm{ml}$ & 0.37 & $1-2 \mathrm{mg} / \mathrm{Kg} / \mathrm{dose}$ \\
Ranitidina & Ampola $50 \mathrm{mg} \mathrm{em} 2 \mathrm{ml}$ & 0.63 & $1-2 \mathrm{mg} / \mathrm{Kg} / \mathrm{dia}$ \\
Penicilina & Frasco 10.000 .000 U em $10 \mathrm{ml}$ & 3.67 & $25-500.000 \mathrm{Ugg} / \mathrm{dia}$ \\
Amicacina & Ampola $100 \mathrm{mg} \mathrm{em} 2 \mathrm{ml}$ & 2.41 & $7,5 \mathrm{mg} / \mathrm{Kg} / \mathrm{dose}$ \\
Midazolan & Ampola $50 \mathrm{mg} \mathrm{em} 3 \mathrm{ml}$ & 4.59 & $0,05-0,2 \mathrm{mg} / \mathrm{Kg} / \mathrm{dose}$ \\
Fentanil & Frasco $500 \mathrm{mcg} \mathrm{em} 10 \mathrm{ml}$ & 2.88 & $2 \mathrm{mcg} / \mathrm{Kg} / \mathrm{dose}$ \\
Ceftriaxone & Frasco $1000 \mathrm{mg} \mathrm{em} 10 \mathrm{ml}$ & 18.90 & $50-100 \mathrm{mg} / \mathrm{Kg} / \mathrm{dia}$ \\
Cefalotina & Frasco $1000 \mathrm{mg} \mathrm{em} 4 \mathrm{ml}$ & 2.23 & $50-100 \mathrm{mg} / \mathrm{Kg} / \mathrm{dia}$ \\
Oxacilina & Frasco $500 \mathrm{mg} \mathrm{em} 3 \mathrm{ml}$ & 2.94 & $100 / 300 \mathrm{mg} / \mathrm{Kg} / \mathrm{dia}$ \\
Ampicilina & Frasco $500 \mathrm{mg} \mathrm{em} 5 \mathrm{ml}$ & 4.81 & $50-400 \mathrm{mg} / \mathrm{Kg} / \mathrm{dia}$ \\
Metronidazol & Frasco $500 \mathrm{mg} \mathrm{em} 100 \mathrm{ml}$ & 3.90 & $7,5 \mathrm{mg} / \mathrm{Kg} / \mathrm{dose}$ \\
\hline
\end{tabular}

A Tabela 4 mostra a apresentação farmacológica, o custo em dólares americanos e a dose pediátrica recomendada por quilograma de peso de cada droga apresentada, segundo referências nacionais ${ }^{(6-7)}$.

\section{DISCUSSÃO}

O número de doses de medicamentos administrados diariamente em crianças, por via intravenosa, neste estudo, foi de 274,9, número superior às 197 doses identificadas em estudo similar ${ }^{(8)}$. Projeção realizada no estudo citado demonstrou uma administração anual de 72.000 doses de medicamentos, enquanto projeção similar neste estudo aponta um total de 98.940 doses. Esse maior número de doses pode ter relação com o tipo de clientela e instituição investigada, também se supõe que demonstra, quantitativamente, a abrangência e importância dessa terapêutica na população pediátrica investigada.

O hospital pesquisado serve de campo de ensino para as áreas de medicina e enfermagem e caracterizase como de atendimento terciário, o que tem correlação com a gravidade do paciente atendido, pois torna-se hospital de referência para elucidação diagnóstica e tratamento de pacientes portadores de enfermidades complexas, que podem necessitar, portanto, de maior diversidade de medicamentos para se obterem os resultados necessários para a cura.

Outro fator a ser considerado advém do tipo de população estudada, a pediátrica, que, por características específicas, requer maior administração de medicamentos por via intravenosa, comparativamente ao adulto, do que por outras vias mais comumente utilizadas como a intramuscular e oral.

Ressalta-se que a massa muscular da criança é, aproximadamente, $38 \%$ menor do que a de um adulto, e a composição corporal muscular de um recém-nascido é de aproximadamente $25 \%$, enquanto que, no adulto, varia em torno de $40 \%$. Devido à pequena massa muscular, poucos sítios são recomendados para injeções intramusculares, também o fluxo sangüíneo muscular irregular pode afetar a absorção de drogas em crianças. Esses fatores minimizam a indicação de administração de medicações por via intramuscular na criança ${ }^{(9)}$. 
Quanto à administração de drogas por via oral em crianças, sabe-se que as menores de seis anos, principalmente, não utilizam medicamentos em cápsula ou comprimidos, devido à dificuldade de deglutição. Algumas dessas drogas são masseradas e rediluídas para administração, porém, comumente, testes específicos não são realizados quanto à validade e qualidade desse procedimento, o que pode corroborar para o aumento da necessidade de se administrarem medicamentos por via intravenosa em crianças ${ }^{(10)}$.

Questiona-se como administrar captopril 0,1 a 0,3mg/Kg em um recém-nascido, quando a apresentação do medicamento varia de comprimidos com $12,5 \mathrm{mg}$ a $100 \mathrm{mg}$ ?

Geralmente, na unidade de pediatria, a enfermeira prepara a diluição líquida desse comprimido. Todavia, desconhece-se a estabilidade do medicamento após a diluição, o período de tempo em que permanece seguro ${ }^{(4)}$ e se essas características apresentam variação, segundo cada tipo de fabricante ou diluente utilizado.

Outro fator a considerar quanto à administração por via oral refere-se ao esvaziamento gástrico mais lento, trato gastrintestinal maior, peristaltismo irregular e secreções gástricas mais alcalinas no recém-nascido, comparado a crianças maiores. Crianças, principalmente recém-nascidos, alimentam-se mais freqüentemente do que adultos, apresentando, conseqüentemente, maior quantidade de alimentos e enzimas digestivas em seu estômago. Esses fatores contribuem para menor absorção de drogas administradas por via oral ${ }^{(9)}$.

Observamos na Tabela 1 que, aparentemente, administrou-se maior quantidade de doses-medicamentos nas unidades de Pediatria Clínica (capacidade de 34 leitos) e UCIP (capacidade de 9 leitos). Porém, ao realizarmos a média de uso de medicamentos por criança, conforme Tabela 2, obtivemos maiores médias na UCIP e Pediatria Cirúrgica (capacidade de 9 leitos).

No que concerne à UCIP, podem ser administrados de seis a quinze preparados farmacêuticos em crianças criticamente enfermas durante o tratamento ${ }^{(11)}$.

Com relação à unidade cirúrgica, atribui-se que a média maior de doses de medicamentos identificada, comparativamente às unidades clínicas (enfermaria e infectologia), pode ter relação com a terapêutica pósoperatória de mais doses de antibióticos e de medicamentos sintomáticos como analgésicos e antieméticos.
Conforme demonstra a Tabela 3, a UCIP possui número bastante maior de profissionais de enfermagem, quando comparada às outras três unidades. Essa constatação tem relação com a maior demanda de cuidados de enfermagem para o atendimento de crianças gravemente enfermas, o que se correlaciona com o aumento da necessidade terapêutica medicamentosa.

Esperava-se obter número maior de profissionais de enfermagem por paciente na unidade cirúrgica $(0,95)$, quando comparada à de infectologia $(1,21)$ e de clínica $(1,20)$, devido ao maior número de doses de medicamentos administrados por via intravenosa e à importante relação existente entre demanda de cuidados de enfermagem na administração da terapia intravenosa em pediatria ${ }^{(1,6-7)}$.

Dentre os 41 tipos de medicamentos administrados por via intravenosa identificados, nenhum possuía apresentação direcionada ao atendimento de necessidades terapêuticas específicas de crianças.

Conforme Tabela 4, nota-se que os medicamentos comercializados em ampolas, como amicacina, furosemida, midazolan e ranitidina, apresentam concentração e dosagem superior à utilizada em cada dose pediátrica, principalmente em crianças até três anos de idade, faixa etária que se caracteriza como a que mais tem tido necessidade de assistência hospitalar, e assim, como todo o excedente da droga deve ser desprezado após a administração, ocorre um considerável aumento no custo do tratamento proposto.

Outro aspecto a considerar, refere-se a drogas como metilpredinosolona e fentanil, cuja concentração por mililitro de solução é muito superior à dose máxima recomendada por dia (Tabela 4).

Por meio.dos dados primários, calculou-se o custo do tratamento medicamentoso de uma criança de $10 \mathrm{Kg}$, que recebia, em 24 horas, doses máximas de metilpredinosolona (uma dose diária), fentanil (dose única), midazolan (dose única), amicacina (duas doses diárias) e metronidazol (três doses diárias). O total de medicamentos administrados foi de US\$6.71, e o total não administrado, isto é, desprezado, foi de US\$39.52.

Esse exemplo ilustra o que ocorre na instituição investigada quotidianamente. Ainda ao custo dos medicamentos, acresce-se o de horas de trabalho de enfermagem na manipulação, durante a diluição, para se reconstituírem algumas drogas, bem como em possível nova diluição durante a administração, necessária à infusão da maioria dos medicamentos, principalmente antibióticos, 
realizada por meio de câmaras de gotejamento (buretas).

Destacamos que, segundo os dados primários, os medicamentos de maior consumo em todas as quatro unidades investigadas foram os antibióticos do tipo amicacina e vancomicina.

Quanto à vancomicina, apesar do custo de U\$13.60 por unidade, devido à comercialização em frasco ampola com apresentação de $500 \mathrm{mg}$, em $5 \mathrm{ml}$ (100mg U\$2.72) e estabilidade pós diluição em refrigeração (4$8^{\circ} \mathrm{C}$ ) de 7 ou 14 dias, conforme recomendação de cada fabricante, pode-se observar menor desperdício do medicamento. Como exemplo, uma criança de $5 \mathrm{Kg}$ receberia, diariamente, $150 \mathrm{mg}$, podendo o excedente ser conservado em refrigerador até a próxima dose.

Entretanto, em relação à amicacina $(100 \mathrm{mg}$ U\$2.41), apesar do menor custo da unidade, observa-se desperdício de medicação a cada dose administrada. A mesma criança receberia três doses diárias, totalizando, em 24 horas, 37,5 mg de amicacina. Como esse antibiótico é comercializado em ampolas de $50 \mathrm{mg} / \mathrm{ml}$, não é possível conservar o medicamento até a próxima dose, acarretando consumo diário de $\bigcup \$ 0.90$ e desperdício de U\$ 1.51. Esse número serve como um alerta da dimensão do problema, visto à grande abrangência do emprego desse antibiótico na assistência, gerando, a cada paciente, um aumento significativo de custos.

Considera-se que a falta de formulações e apresentações de medicamentos especificamente direcionados à faixa etária pediátrica, além de preocupante, constitui um desafio na busca de qualidade da assistência de enfermagem prestada à criança.

Os esforços, porém, não são apenas da enfermagem, mas, sim de outros profissionais de saúde, como médicos e farmacêuticos.

Em alguns hospitais, uma estratégia criada para diminuir os impactos desse problema, refere-se à criação de centrais de preparo e dispensa de medicamentos. Essa centralização de preparo promove uma utilização mais racional de medicamentos em hospitais.

Com uma abrangência mais ampla para tentar solucionar a falta de formulações pediátricas de medicamentos, o Food and Drugs Administration - FDA ${ }^{(3)}$, dos Estados Unidos da América, propõe questionamentos para a criação de novas regulamentações, com o objetivo de obrigar a indústria farmacêutica a alcançar maior segurança e eficácia de certas drogas novas utilizadas em pediatria. O FDA levantou importantes questionamentos, ainda em discussão, e que deveriam, igualmente, fazer parte de diretrizes de trabalhos realizados por aqueles que regulamentam essa área da saúde em nosso país.

Como exemplo de alguns dos questionamentos levantados, temos ${ }^{(3)}$ :

- Quando a elaboração e comercialização de drogas para a pediatria devem ser impostas à indústria farmacêutica? - O custo da elaboração das formulações pediátricas justifica a desistência de comercialização?

- O número de pacientes que utilizam determinada droga deve influenciar o desenvolvimento de uma formulação pediátrica?

- A indústria farmacêutica deve ser obrigada a alocar recursos para desenvolver uma formulação pediátrica?

- A aprovação de novas drogas para adultos deve ser protelada até que a formulação pediátrica esteja pronta para comercialização?

- O que deve ser considerado, como esforços razoáveis da indústria farmacêutica, para acatar solicitações ou regulamentações governamentais?

Assim, o presente estudo tem também por finalidade estimular profissionais da área da saúde, os quais cuidam de crianças hospitalizadas, bem como outros envolvidos na área de assistência médica, quanto à necessidade de discussões freqüentes sobre o tema $\mathrm{e}$ reivindicações junto à indústria farmacêutica e órgãos governamentais, quanto à pertinência e importância de se atenderem às características terapêuticas pediátricas de utilização de medicamentos administrados por via intravenosa.

Igualmente relevante é o acompanhamento das discussões que começam a aparecer em literaturas internacionais, principalmente norte-americanas, a respeito do alcance das propriedades terapêuticas esperadas em medicamentos administrados por via intravenosa em crianças, sem necessidade de custo excessivo, bem como de risco de complicações decorrentes da apresentação inadequada.

\section{CONCLUSÕES}

Nas quatro unidades pediátricas estudadas, em trinta dias, foram administradas 8245 doses de medicamentos por via intravenosa, com média diária de 274,83 , e projeção anual de 98.940 doses. 
Os medicamentos mais administrados por via intravenosa em crianças foram: metilpredinosolona, vancomicina, furosemida, ranitidina, penicilina, amicacina, midazolan, fentanil, ceftriaxone, cefalotina, oxacilina, ampicilina e metronidazol.

A totalidade, 41, dos medicamentos identificados não tinha apresentação farmacológica pediátrica.
O custo estimado da terapia medicamentosa intravenosa em uma criança, em 24 horas, foi de $\bigcup \$ 46.23$, sendo que $\cup \$ 6.71$ de medicamentos foram administrados e \$ 39.52 desprezados, por excederem as necessidades terapêuticas diárias da criança. Observou-se que a comercialização em ampolas também é fator que contribui para o aumento indireto dos custos.

\section{REFERÊNCIAS BIBLIOGRÁFICAS}

1. Bohony J. 9 Common IV complications and what do about them. Am J Nurs 1993; 93(10):45-9.

2. Rimar JM. Guidelines for the intravenous administration of medications used in pediatrics. MCN 1982; 7:184-97.

3. Nahata MC. Pediatric drug formulations: challenges and potentials solutions. Ann Pharmacother 1999; 33:247-9.

4. National Institute of Child Health and Human Development. NIH News Alert. Washington: National Institute of Health; 1997.

5. Kuhn MM. Intravenous Therapy. In Kuhn MM. Pharmacotherapeutics: a nursing process aproach. $4^{\underline{a}}$ ed. Philadelphia: F A Davis; 1998. p. 1033-50.

6. Pignatari AC. Fomulário Terapêutico. São Paulo: UNIFESPSPDM; 2000.

7. Carvalho WB, Fascina L, Moreira G. Manual de Terapia Intensiva Pediátrica. São Paulo: Atheneu; 1994. p: 450-67. 8. Hockley B, Samuel D, Pratt M. Audit pinpoints IV drug administration pitfalls. J Intrav Nurs 1997; 20(8):287-8.

9. Pedreira MLG. Uso de bombas de infusão na terapia intravenosa em crianças assistidas em unidades de cuidados intensivos pediátricos: contribuições para estudos clínicos e técnicos. [Tese]. São Paulo: UNIFESP-EPM; 1999. 10. Nahata MC. Lack of pediatric drug formulations. Pediatrics 1999; 104(3):607-9.

11. Carvalho WB. Farmacologia clínica e medicamentosa: um assunto preocupante. Medicus 1996; 3:11-26. 